\title{
THE HYDRAULIC AND ELECTRICAL FRACTAL DIMENSION OF REGOLITH
}

\author{
Tania Dhu, Graham Heinson \& John Joseph
}

CRC LEME, School of Earth and Environmental Science, University of Adelaide, Adelaide, SA, 5005

\section{INTRODUCTION}

Electromagnetic (EM) induction techniques have been employed in numerous environmental and exploration situations including groundwater investigations, unexploded mine ordinance and nickel-sulphide exploration. The Earth's EM response is a diffusion process that is dependent upon electrical resistivity. For piecewise smooth media this process is described by Maxwell's equations.

Everett \& Weiss (2002) show that it is not appropriate to consider the EM response of the sub-surface as essentially uniform with a Gaussian random noise envelope. In other words, the sub-surface cannot be considered to be essentially homogeneous with Gaussian random noise superimposed by geological structure. Instead, Everett \& Weiss demonstrated that the EM response has a correlated scale-length that is a function of the heterogeneity of the sub-surface. That is, the resistivity of the sub-surface is a function of the dimension, or scale-length, over which it is measured. A fractal dimension can describe such scale-length dependence of resistivity. Everett \& Weiss conducted two surveys: one over a fractured sandstone aquifer; and one over a floodplain using a Geonics Ltd. EM34-3 ground conductivity meter. Noise in both data sets was found to be repeatable, implying that it was not from instrumental or atmospheric effects. Both data sets were fractal in nature representing rough, self-similar media rather than smoothly varying phenomena.

The scale-length dependence (described by the fractal dimension) of EM measurements is an alternative and potentially useful approach to interpreting ground and airborne data. For example, regions of clay and saline sediments may have the same range of resistivities (typically 1-10 $\Omega \mathrm{m}$ ), making it hard to distinguish between such sources. However, they may well have a different noise characteristic as determined from the fractal dimension. Additionally, in fractured rock environments the fractal-dimension of the EM data may relate to the power-law connection of fractures. As hydraulic conductivity also exhibits a fractal scaling, the ultimate aim of the project is to attempt to link electrical and hydraulic pathways in a novel manner.

In this abstract, a preliminary study has been carried out on raw TEM signal strength from a ground survey gathered at Tunkillia, South Australia, with comparison to a 1D inversion section.

\section{FRACTAL DIMENSION}

Many of the shapes found in nature are very irregular and hard to define via classical geometry, hence the use of fractals as a means of representing these shapes. Fractals are sets that are self-similar-they are the same (either statistically or exactly) on all scales. The fractal dimension is then a method of quantifying the complexity of that shape.

Calculation of the fractal dimension via power spectral density (PSD) plots can be used to determine between EM responses that have Gaussian random-noise (signals with no spatial-correlation), and non-Gaussian fractal signals with a spatial-correlation. When the fractal dimension lies between -1 and 1 the signal is considered to be noise, whereas values between 1 and 3 (or -1 and -3) are non-Gaussian. Higher values correspond to greater long-range correlation within the signal.

A true fractal is infinitely self-similar, in other words, repeatable on all scales. Obviously this is not the case in the real world where many phenomena are prefractal- they exhibit fractal properties over a limited scale. Due to this constraint, numerous papers have considered a stationarity threshold (e.g., Fardin et al. 2001) that is used to define the scale over which a media is fractal. Considering the fractal dimension of a data set outside of this window leads to a dimension that is not a true representative of the media and will tend to change as larger or smaller regions are considered rather than remaining constant.

\section{SURVEY TECHNIQUE}

A survey was conducted at Tunkillia, in the Gawler Craton, South Australia, over the northern end of the Yarlbrinda Shear Zone. Calcrete sampling in the region revealed a gold anomaly of $25 \mathrm{~km}^{2}$, the largest in Australia. The area is covered by extensive sand dunes that have hindered exploration in the region. 
A ground TEM survey was conducted with a NanoTEM transmitter and GDP16 receiver system from Zonge Engineering and Research Organisation. A 20 m square transmitter configuration was used, with a centrally located square $5 \times 5 \mathrm{~m}$ receiver. Stations were separated by $20 \mathrm{~m}$ and data were collected at 110 stations covering $4.3 \mathrm{~km}$. Data were inverted using STEMINV (MacInnes et al. 2001), a smooth-model inversion technique, and a section is shown in Figure 1.

\section{METHOD}

Fractal dimension calculations were performed on the raw TEM signal strength data to avoid any smoothing processes inherent in the inversion processes. The raw signal strength was divided into 31 time windows, ranging from $0.00122 \mathrm{~ms}$ through to $3.025 \mathrm{~ms}$, with later time values corresponding to greater depths. Fractal dimensions were calculated from the PSD plotted on a log-log graph by fitting a one-dimensional line by least squares. The slope of this line of best fit is then equal to the fractal dimension.

A stationarity threshold was calculated by computing the fractal dimension of different size portions of the data. Data were divided into bins of increasing size, from $2 \%$ up to $100 \%$ (using $2 \%$ intervals) and the fractal dimension for each bin plotted on a graph. Three ways of binning the data were used, one always using the left-most point, one always using the right-most point and one that always contained the central point

Once a stationarity threshold was determined the fractal dimension was calculated using a moving window. The fractal dimension of the first 33 points was calculated and plotted above the central point, then the window was moved up one so that points 2-34 were binned, then 3-35 etc. and the fractal dimensions calculated and plotted.

\section{RESULTS AND DISCUSSION}

The fractal dimension for the entire 110 data points was calculated, as shown in Figure 2. Results concurred with the experimental outcomes presented by Everett \& Weiss (2002). The fractal dimension changes marginally between time windows, but for the majority it lies between 1.6 and 1.8. When inverting data the late-time windows were not included as they are considered to no longer be recording the transmitted signal but just random noise. We find that the fractal dimension of such late-time windows was less than 1, implying that they are random noise.

Stationarity threshold calculations are shown in Figure 3. Three methods were used; taking data from the lefthand side of the profile; from the centre; and from the right-hand side. All three methods of binning returned similar results, implying that the fractal dimension calculations were primarily dependent upon the number of data points and size of data set. Figure 4 shows the fractal dimension calculated across the data set for two different time windows. There are correlations between regions of high resistivity in the section in Figure 1, and low fractal dimension however the occurrences of these seem to be displaced somewhat to the right.

\section{CONCLUSIONS}

We have tentatively begun to show the relationship between scale-length dependence of EM responses and the electrical resistivity properties of the Earth. As resistivity is largely dependent upon hydraulic parameters (porosity, tortuosity, permeability) and with clay content, the aim of the project is to examine the effect of hydraulic conductivity as a function of EM response.

For further inferences to be drawn the experiment needs to be re-examined using a more complete data set and preferably benchmarked against geological variations collected via another technique. Thus, further field studies are underway to examine much larger data sets collected with smaller site spacings over regions of known soils and bedrock at Jamestown, South Australia and with the towed TEM surveys of Barrett et al. (2003) along the Murray River. The airborne data sets from Gilmore will also be examined at some stage.

\section{REFERENCES}

BARRett B.E., Heinson G.S., HAtch M. \& Telfer A. in press. A water-borne transient electromagnetic system for river salt-load detection. Ground Water.

EveretT M.E. \& Weiss C.J. 2002. Geological noise in near-surface electromagnetic induction data. Geophysical Research Letters 29(1), p. 1010.

FARDin N., STEPHANSSON O. \& JING L. 2001. The scale dependence of rock joint surface roughness. International Journal of Rock Mechanics and Mining Sciences 38(5), 659-669.

MACINNESS S. \& RAYMOND M. 2001. STEMINV Documentation - Smooth-Model TEM Inversion, version 3.00. Zonge Engineering and Research Organisation Inc. 

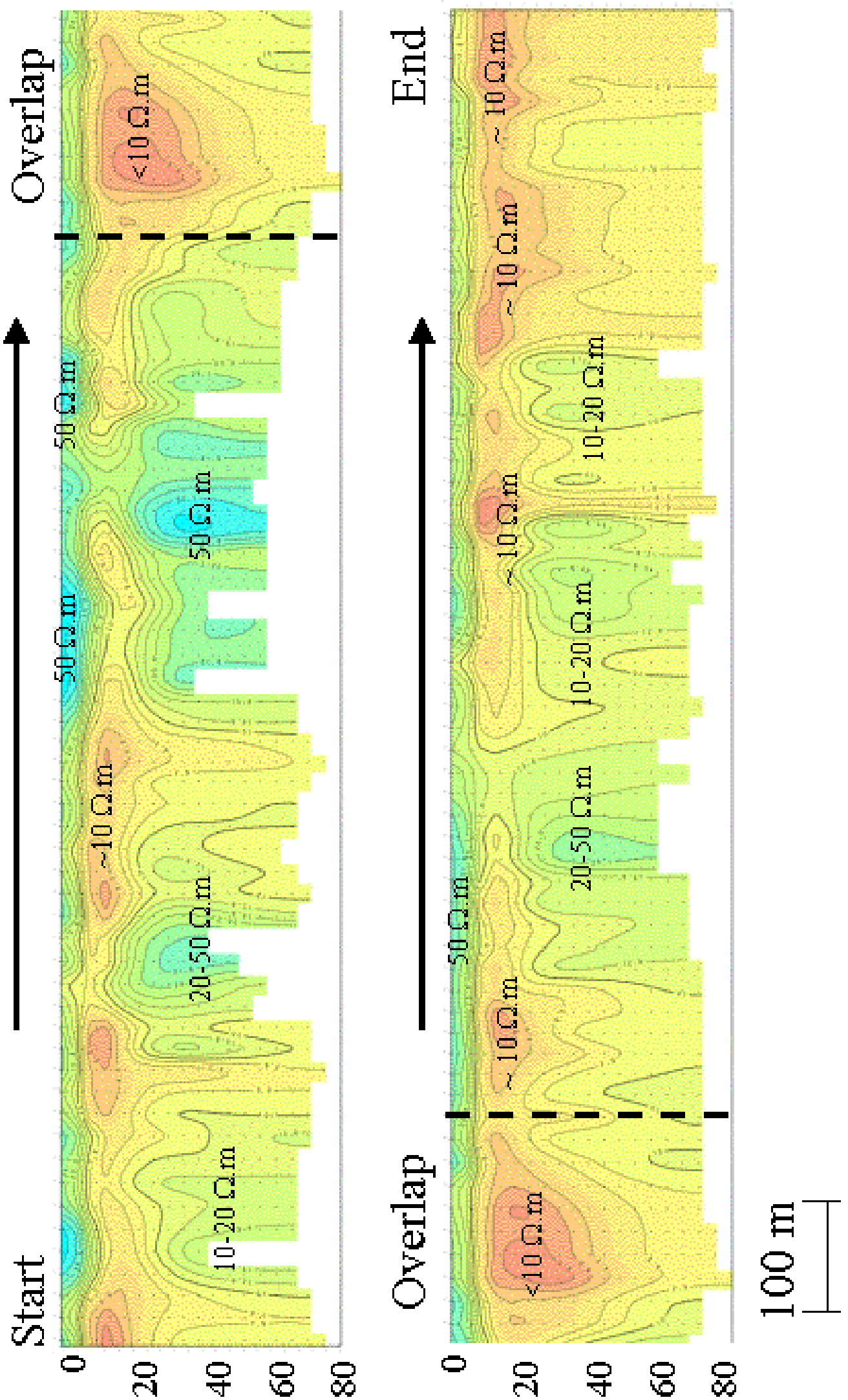

Figure 1: Two-dimensional inversions of TEM data from Tunkillia. The plot is split into two profiles, with a 200 m overlap section. 

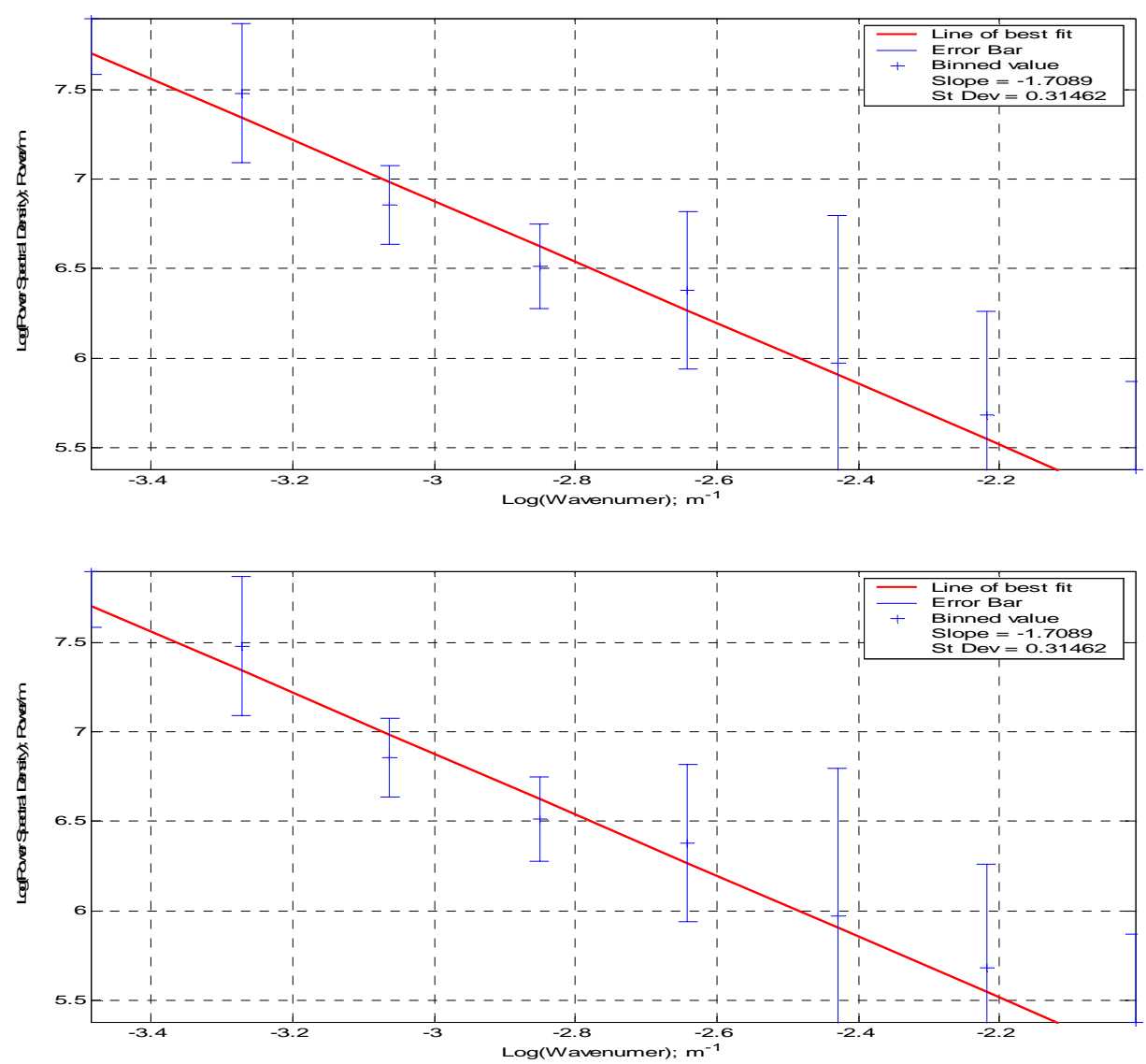

Figure 2: PSD plots for time windows 4 (top), 8 (second down),12 (third

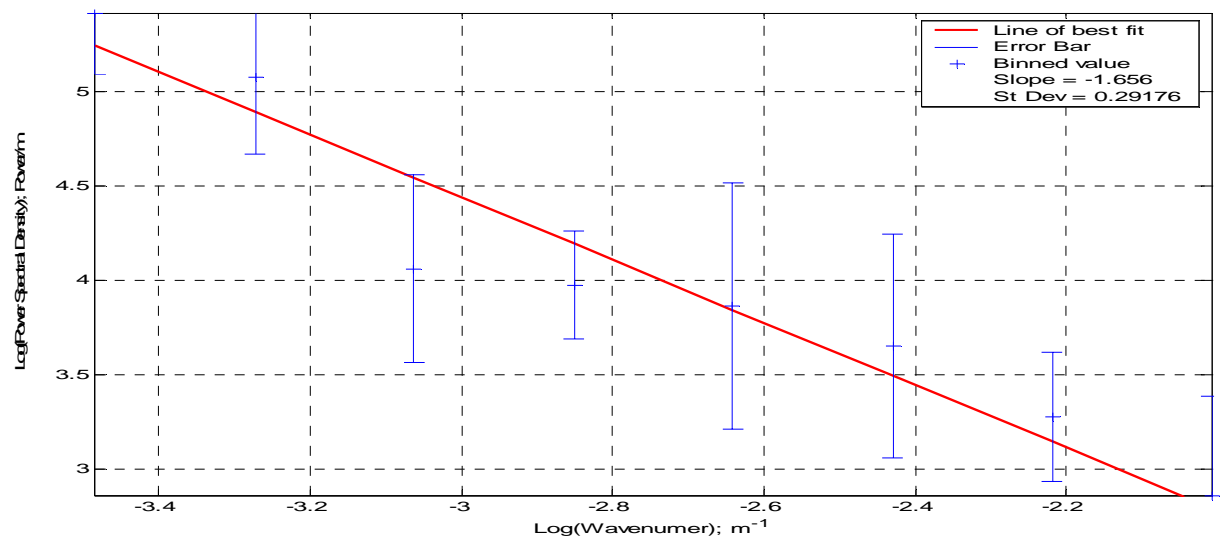
down), and 31 (bottom) across all 110 points.

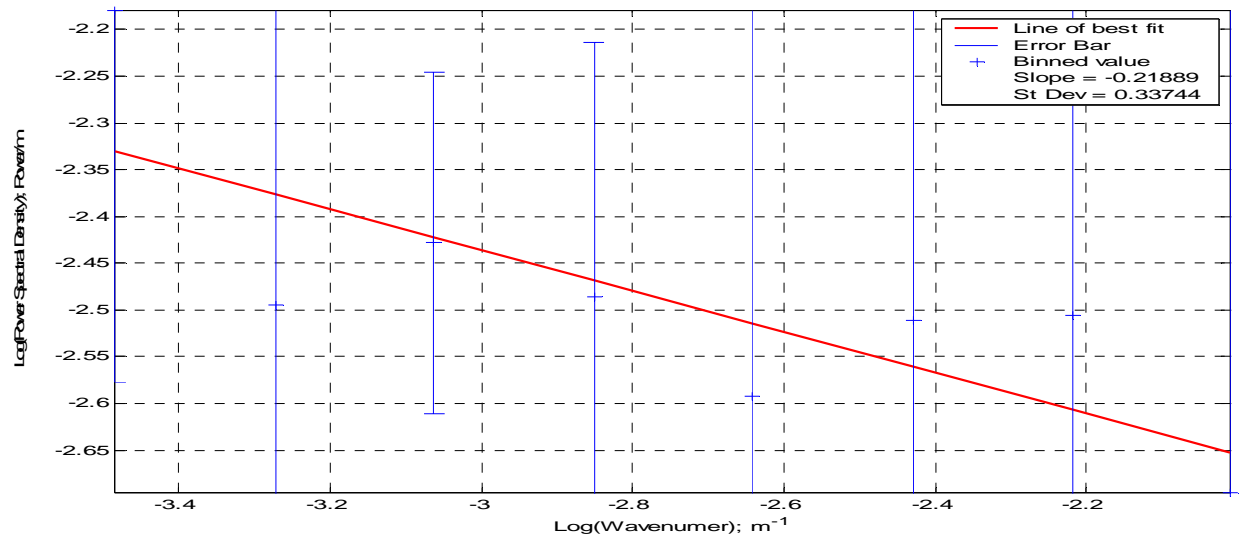




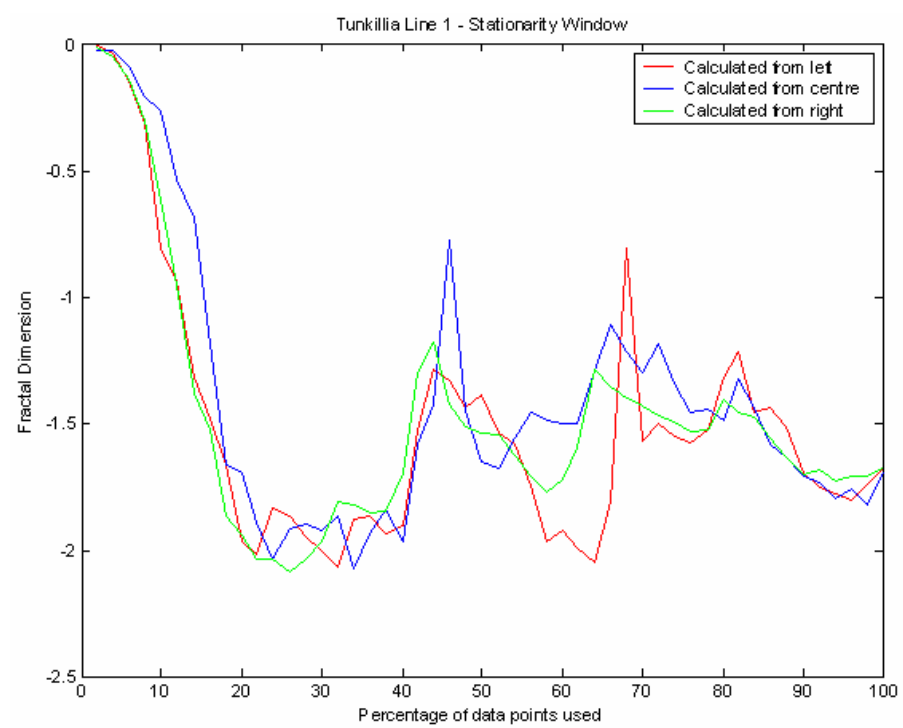

Figure 3: Stationarity threshold calculations (time window 2).
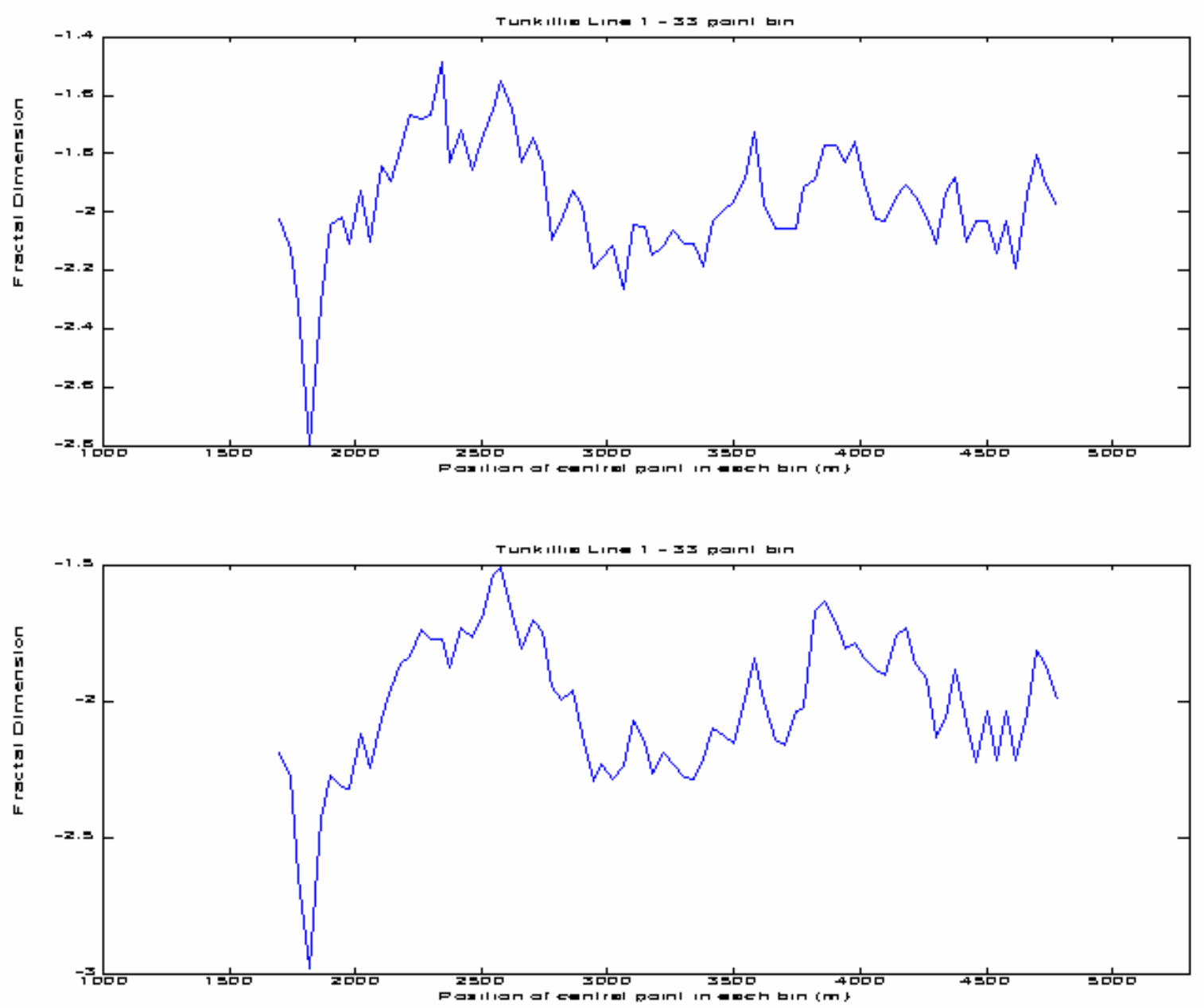

Figure 4: Fractal dimension using 33 point moving window for time window 4 (top) and 8 (bottom) 